\title{
RECONSTRUCTION OF BUILDING FOOTPRINTS USING SPACEBORNE TOMOSAR POINT CLOUDS
}

\author{
M. Shahzad ${ }^{\text {a }}$, X. X. Zhu ${ }^{\text {a,b* }}$ \\ ${ }^{a}$ Helmholtz Young Investigators Group "SiPEO", Technische Universität München, 80333 Munich, Germany - \\ muhammad.shahzad@bv.tum.de \\ ${ }^{\mathrm{b}}$ Remote Sensing Technology Institute (IMF), German Aerospace Center (DLR), Oberpfaffenhofen, 82234 Weßling, Germany - \\ xiao.zhu@dlr.de
}

\section{Commission VI, WG VI/4}

KEY WORDS: TerraSAR-X/TanDEM-X, tomographic SAR (TomoSAR) inversion, 3-D point cloud, building footprint, clustering

\begin{abstract}
:
This paper presents an approach that automatically (but parametrically) reconstructs 2-D/3-D building footprints using 3-D synthetic aperture radar (SAR) tomography (TomoSAR) point clouds. These point clouds are generated by processing SAR image stacks via SAR tomographic inversion. The proposed approach reconstructs the building outline by exploiting both the roof and façade points. Initial building footprints are derived by applying the alpha shapes method on pre-segmented point clusters of individual buildings. A recursive angular deviation based refinement is then carried out to obtain refined/smoothed 2-D polygonal boundaries. A robust fusion framework then fuses the information pertaining to building façades to the smoothed polygons. Afterwards, a rectilinear building identification procedure is adopted and constraints are added to yield geometrically correct and visually aesthetic building shapes. The proposed approach is illustrated and validated using TomoSAR point clouds generated from a stack of TerraSAR-X high-resolution spotlight images from ascending orbit covering approximately $1.5 \mathrm{~km}^{2}$ area in the city of Berlin, Germany.
\end{abstract}

\section{INTRODUCTION}

Modern very high resolution (VHR) spaceborne SAR sensors such as TerraSAR-X/ TanDEM-X and COSMO-SkyMed can deliver data beyond the inherent spatial scales of buildings. These VHR data are particularly suited for detailed urban mapping. Among advanced SAR interferometric methods, TomoSAR is the most general 3-D SAR imaging principle, because of its layover separation capability. Geocoding high density of scatterers, retrieved from TomoSAR, into world coordinates enable the generation of high quality TomoSAR point clouds, containing not only the $3 \mathrm{D}$ positions of the scatterer location but also estimates of seasonal/temporal deformation, that are very attractive for generating 4-D city models from space.

Object reconstruction from spaceborne TomoSAR point cloud has been recently started (D'Hondt et al., 2013)(Shahzad and Zhu, 2015a) (Fornaro et al., 2014). These point clouds have point density in the range of $600,000 \sim 1,000,000$ points $/ \mathrm{km}^{2}$ and are associated with some characteristics that are worth to mention (Zhu and Shahzad, 2014):

1) TomoSAR point clouds deliver moderate $3 \mathrm{D}$ positioning accuracy on the order of $1 \mathrm{~m}$;

2) Few number of images and limited orbit spread render the location error of TomoSAR points highly anisotropic, with an elevation error typically one or two orders of magnitude higher than in range and azimuth (Zhu and Bamler, 2012);

3) Due to the coherent imaging nature, temporally incoherent objects such as trees cannot be reconstructed from multipass spaceborne SAR image stacks;

4) TomoSAR point clouds possess much higher density of points on the building façades due to side looking SAR geometry enabling systematic reconstruction of buildings footprint via façade points analysis.

As depicted over smaller and larger areas in (Zhu and Shahzad, 2014) and (Shahzad and Zhu, 2015a), façade reconstruction turns out to be an appropriate first step to detect and reconstruct building shape from these point clouds when dense points on the façade are available. Especially, when data from multiple views, e.g., from both ascending and descending orbits, are available, the full shape of buildings can be reconstructed using extracted façade points. However, there are cases when no or only few façade points are available. This happens usually for lower height buildings and renders detection of façade points/regions very challenging. Moreover, problems related to the visibility of façades mainly pointing towards the azimuth direction can also cause difficulties in deriving the complete structure of an individual building. These problems motivate researchers to derive full 2-D building footprint via roof point analysis. In this regard, based on different object contents illuminated by side looking SAR, the following three cases could be derived using data acquired from one incidence angle e.g., image stacks from ascending orbit only.

Case 1: Higher density of façade points present with no or very few roof points - In this case, the complete 2D/3D building shapes could be fully reconstructed by adding points from multiple incidence angles. The solution to this case is demonstrated in (Shahzad and Zhu, 2015a) where 3-D façades model have been reconstructed for high rise buildings using one incidence angle only and in (Zhu and Shahzad, 2014) where full shape of the building was derived by prior fusion of two point clouds from ascending and descending stacks.

Case 2: Higher density of façade points present together with existence of relatively high density of roof points - This case allows to reconstruct full shape of the building footprints from a

Corresponding author 
single data stack by making use of both façade and roof points. Thus, the side of the building visible to the sensor could be reconstructed as the first step and later the other side of the building could be completed by exploiting the available roof points.

Case 3: No or very few façade points available but enough roof points exist - This case particularly appears for lower height buildings rendering detection of façade points/regions very challenging. This motivates us to obtain the full 2-D/3-D footprint of these buildings via roof point analysis only using conventional techniques as applied by LiDAR community. Even though these techniques are very much matured, still their adaptation to TomoSAR point clouds is not straight forward due to different object contents illuminated by side looking SAR together with problems related to low and varying point density and much lower positioning accuracies of TomoSAR point clouds in comparison to airborne LiDAR.

In this paper, we propose a novel data driven approach that systematically allows automatic reconstruction of 2-D/3-D building footprints using unstructured TomoSAR points clouds generated from one incidence angle only. The approach proposes new methods and aims at finding a more general and systematic solution towards automatic reconstruction of the whole city area. The paper essentially presents solutions for the latter two cases (i.e., case 2 and case 3 ) by extending (or utilizing) the solution provided for case 1 in (Shahzad and Zhu, 2015a). Following are the innovative contributions proposed in this paper:

1) A recursive angular deviation based approach is presented to smooth/refine the initial coarse building polygons obtained using alpha shapes (generalization of convex hulls).

2) A novel façade-roof fusion procedure is proposed which is robust and fuses the legitimate Façade-Polygon pair together by interpreting the refined/smoothed polygon of each building as a graph.

3) An effective and robust procedure is developed for rectilinear identification of building polygons.

4) Finally, due to the high inclination angle of the TerraSAR$\mathrm{X}$ orbit, i.e. near-polar orbit, the approach presented in (Shahzad and Zhu, 2015a) may fail to reconstruct building façades facing North or South due to lack of measurements. This paper inherently provides solution to this problem by exploiting roof points in determining the complete shape/footprint of the building.

\section{METHODOLOGY}

The whole processing begins by first identifying the probable building regions. This is accomplished by incorporating information pertaining to façades as prior knowledge (i.e., regions corresponding to higher point density indicate probable façade regions). Thus, building façade points are extracted, segmented to points belonging to individual façades, and further reconstructed. The reconstructed façades are used to select seed points which are used to extract available roof points via a minimum height constraint surface normals based region growing procedure. And then roof points without the support of façades point (i.e., case 3) are further extracted out from the remaining points by formulating the extraction problem into an energy minimization framework. Detailed procedure of reconstructing façades and extracting building points via hybrid region growing and energy minimization procedure are described in (Shahzad and Zhu, 2015a)(Zhu and Shahzad, 2014) and (Shahzad and Zhu, 2015b) respectively.

In this work, automatic segmentation of previously detected/extracted building points is then obtained by clustering points belonging to individual buildings. Later, boundary/outline polygons (or footprints) are reconstructed and refined/smoothed for each individually segmented building cluster. Afterwards, robust fusion of legitimate Façade-Polygon pairs is carried out to improve geometrical accuracy of the refined footprints. Finally, after identification of rectilinear footprints, rectangular constraints are inserted to yield geometrically correct and visually aesthetic building shapes. Next we explain the whole procedure in detail.

\subsection{Segmentation into individual buildings}

The extracted building points are segmented into individual clusters such that each cluster represents points from an individual building using the concept of density connectivity (Ester et al., 1996). I.e., two points are considered to be directly density connected to each other if one is in the neighborhood vicinity of the other point. If the two points are not directly connected to each other, still they can be density connected to each other if there is a chain of points between them such that they all are directly density connected. Thus starting from a point, all points that are density connected to each other are clustered into single group representing an individual building. These clustered points are then removed and the procedure is repeated for remaining number of points until all the points are assigned to any one particular cluster.

\subsection{Coarse building footprint}

Reconstruction of building shape is initially obtained by employing alpha shapes (generalization of convex hull) around each segmented building (Edelsbrunner and Mücke, 1994). The output of the alpha shape (or $\alpha$-shape) algorithm is the vertices that describe the 2-D polygonal boundary of the building footprint. The reconstructed shape depends on a particular value of $\alpha$ which has to be carefully chosen since $\alpha$ controls the model complexity. An overlarge $\alpha$ could make it difficult to follow concave polygonal shapes, e.g., an L-shaped building. Therefore, an estimate of $\alpha$ that produces reliable building shape, including smaller structures, may be chosen as twice of the mean of Euclidean distances computed for every point from its nearest neighbor among the set of building points (Dorninger and Pfeifer, 2008).

\subsection{Refined outlines}

Alpha shapes method provides good initial estimates of building outlines. However, due to lower point density of TomoSAR points, it only defines the coarse outline of an individual building. The resulting polygons are therefore irregular and need to be refined/ regularized. If we denote $V_{\text {alpha }}=\left\{\mathbf{V}_{i=1, \ldots, N}\right\}$ as a set containing $N$ matrices of building polygons returned by the alpha shapes algorithm and $\mathbf{V}_{j}$ with $(j \in i)$ as the matrix containing 2-D vertices of the initial alpha polygon of the $j$ th building, then inspired from (Dorninger and Pfeifer, 2008), the recursive procedure summarized in Table 1 is adopted to refine the coarse reconstructed building footprints returned by the alpha shapes algorithm. 


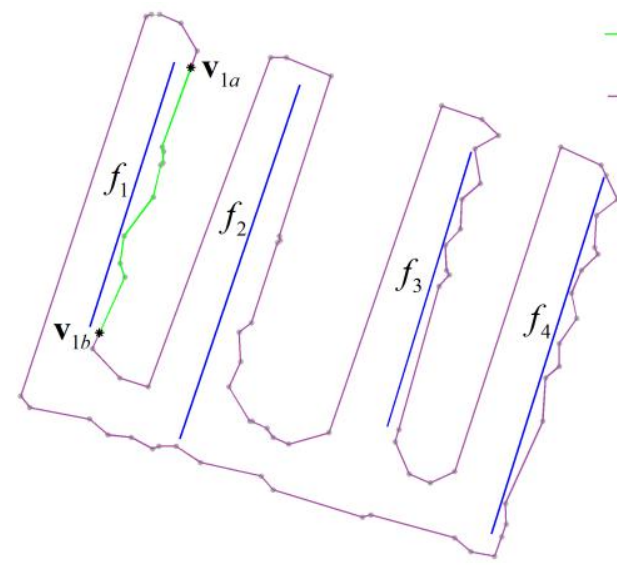

(a)

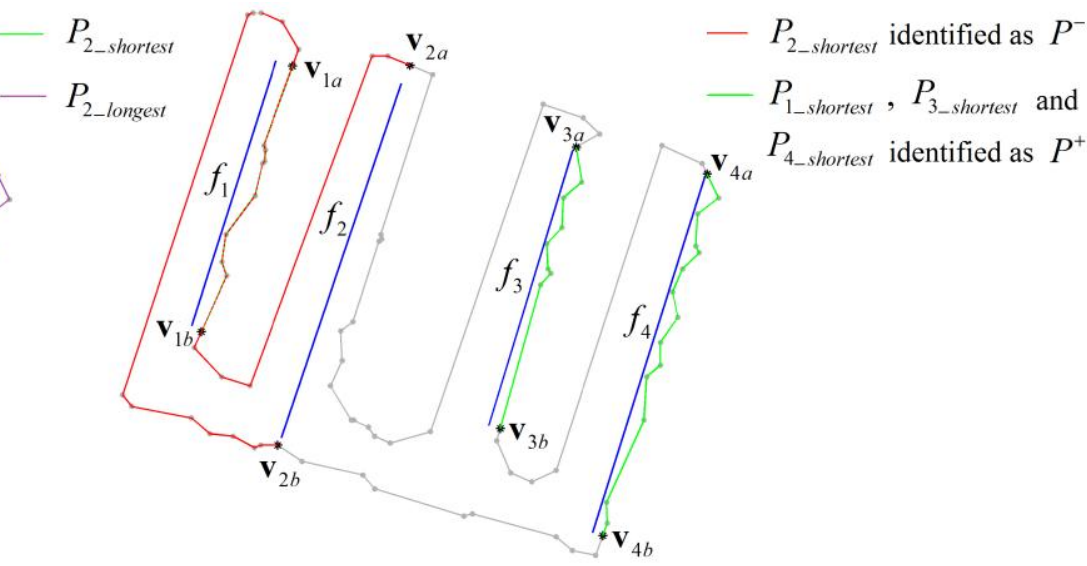

(b)

Figure 1: Illustration of the concept of $P_{t_{-} \text {shortest }}$ and $P^{+}$. Refined polygon of one particular $j$ th building $\tilde{\mathbf{V}}_{j}$ having vertices $\mathbf{v}_{k=1, \ldots, m}$ with four reconstructed façades $f_{1}, f_{2}, f_{3}$ and $f_{4}$ that are to be incorporated. (a) illustrates the concept of shortest and longest paths associated for a particular façade $f_{1} \cdot \mathbf{v}_{1 a}$ and $\mathbf{v}_{1 b}$ denotes the closest points on the polygon/graph $\tilde{\mathbf{V}}_{j}$ to the two endpoints of façade $f_{1}$ respectively; (b) Illustration of the concept of positive path $P^{+} . P_{2_{-} \text {shortest }}$ is identified as $P^{-}$as there exist points in $\mathbf{K}_{2}$ that are also present in $\mathbf{P}_{2_{-} \text {shortest }}$ i.e., $\mathbf{K}_{2} \cap \mathbf{P}_{2_{-} \text {shortest }} \neq \varnothing$ since $\mathbf{P}_{2_{-} \text {shortest }}$ contains points on red line and

$$
\mathbf{K}_{2}=\left\{\mathbf{v}_{1 a}, \mathbf{v}_{1 b}, \mathbf{v}_{3 a} \mathbf{v}_{3 b} \mathbf{v}_{4 a} \mathbf{v}_{4 b}\right\} \text {. }
$$

Given: Alpha shape polygon of $j$ th building $\mathbf{V}_{j}$.

1. Initialize: $\mathbf{V}_{\text {prev }}=\mathbf{V}_{j}$

\section{2. while (1)}

3. Compute angular deviation matrix $\beta$

4. Identify indices in $\boldsymbol{\beta}$ greater than $\theta_{\text {ang }}$

5. Extract vertices that corresponds to the identified indices in $\mathbf{V}_{\text {prev }}$ and assign them to $\mathbf{V}_{\text {new }}$

6. if number of elements in $\mathbf{V}_{\text {prev }}=$ number of elements in

$$
\mathbf{V}_{\text {new }}
$$

7. break

8. else

9. $\quad \mathbf{V}_{\text {prev }}=\mathbf{V}_{\text {new }}$

10. end if

11. end loop

12. $\tilde{\mathbf{V}}_{j}=\mathbf{V}_{\text {new }}$

Table 1: Refinement of alpha shape polygons.

The procedure in begins by computing the angular deviations at each vertex point of the alpha polygon as

$$
\beta_{j}=\left\{\begin{array}{ll}
\theta_{j} & \text { if } \theta_{j} \leq 90^{\circ} \\
180-\theta_{j} & \text { if } \theta_{j}>90^{\circ}
\end{array} \text { with } \theta_{j}=\cos ^{-1}\left(\frac{\mathbf{d v}_{j} \cdot \mathbf{d v}_{j+1}}{\left\|\mathbf{d v}_{j}\right\|\left\|\mathbf{d v}_{j+1}\right\|}\right)\right.
$$

where ' ' denote the dot product and $\mathbf{d} \mathbf{v}_{j}$ is the direction vector computed at each edge formed by connecting two consecutive vertices $\mathbf{v}_{j}$ and $\mathbf{v}_{j+1}$ of the polygon $\mathbf{V}_{\text {prev }}$ (initialized to $\mathbf{V}_{j}$ ). Step 4 and 5 ensures that all those vertices (or edges) having angular deviations less than the threshold $\theta_{\text {ang }}$ are removed.

$\mathbf{V}_{\text {new }}$ and $\mathbf{V}_{\text {prev }}$ are then compared and the process repeats itself if any vertex is removed in the current recursive iteration i.e.,
$\mathbf{V}_{\text {new }}$ and $\mathbf{V}_{\text {prev }}$ do not contain the same number of elements. Finally, the process terminates when there is no further removal of vertices.

\subsection{Incorporating reconstructed façades}

To improve the geometrical accuracy of the footprints, the reconstructed façades are fused with the refined building polygons. For this purpose, the façade associated to each refined building polygon is categorized into following two types:

1) Type I façade: Façade fully or partly inside the refined polygon.

2) Type II façade: Façade lying completely outside but associated to the refined polygon.

The above mentioned two façade types are fused with the refined building polygon in slightly different manners as explained later.

\subsubsection{Identification of legitimate Façade-Polygon pairs}

In order to achieve fusion of reconstructed façades with the refined building polygons, the foremost task is to identify the association of each façade to its respective building polygon.

Identification of type I façades is easily achieved by checking if the endpoints of the reconstructed façades lie inside the polygon. Thus if both or at least one of the façade endpoints lie inside the polygon, it is categorized to be type I façade.

To identify façades of type II, following procedure is adopted:

1) First the midpoint of the reconstructed façade is computed and two points are chosen in opposite directions orthogonal to the reconstructed façade at a distance $d$ from the midpoint; 
2) Compute intersections of line $_{1}$ and line $_{2}$ with all the building polygons. Here line ${ }_{1}$ denotes the line segment formed from by connecting midpoint to one of the chosen points and similarly line ${ }_{2}$ is line segment formed by connecting midpoint to the other opposite point;

3) If there exists an intersection of line or $_{\text {line }}$ with any of the building polygons, façade is assigned to the polygon with which the intersection occurs. In case there are more than one line-polygon intersections or both line ${ }_{1}$ or line 2 intersects with different polygons, the façade is assigned to the polygon having the intersection point nearest to it.

Implementation-wise, steps 1-3 are performed in a recursive manner. I.e., $d$ is initialized to $1 \mathrm{~m}$ and steps 1-3 are carried out. In case, there exists no line-polygon intersection (i.e., façade is not assigned to any polygon), the procedure repeats itself but this time $d$ is incremented by $1 \mathrm{~m}$. The recursion stops if either the façade is assigned to any polygon or the distance $d$ exceeds a certain threshold which is set to fixed $20 \mathrm{~m}$ in this work. Thus a façade is only associated/paired to any building polygon if it lies at a distance less than $20 \mathrm{~m}$, otherwise it is regarded to have no polygon associated to it (i.e., categorized to case 1).

\subsubsection{Fusion of Façade-Polygon pairs}

Similar to earlier notation, let us denote $V_{\text {refined }}=\left\{\tilde{\mathbf{V}}_{i=1, \ldots, N}\right\}$ as a set containing $N$ matrices of refined building polygons with $\tilde{\mathbf{V}}_{j}=\left\{\mathbf{v}_{k=1, \ldots, m}\right\}(j \in i)$ being the matrix containing 2-D vertices of the $j$ th refined polygon having $m$ vertices and $f_{r=1, \ldots, s}$ as the corresponding $s$ number of (paired) reconstructed façades. Now if the building polygon, formed by connecting vertices of $\tilde{\mathbf{V}}_{j}$, is interpreted as a graph, then we may define a path $P_{t}$ for any particular façade $f_{t}$ as a way consisting of polygonal chain of vertices that connect two points $\mathbf{v}_{t a}$ and $\mathbf{v}_{t b}$ lying on the graph/polygon. I.e., the polygonal segment comprising of all the points of the polygon $\tilde{\mathbf{V}}_{j}$ within the interval $\left[\mathbf{v}_{t a} \mathbf{v}_{t b}\right]$ defines path $P_{t} \cdot \mathbf{v}_{t a}$ and $\mathbf{v}_{t b}$ denote points on the building polygon which are nearest to the two endpoints of the particular reconstructed façade $f_{t}$. Since in our case, the polygon is non-intersecting (or simple), it renders only two distinct paths to exist, referred as $P_{t_{-} \text {shortest }}$ and $P_{t_{-} \text {longest }}$ (see Figure 1). If the path length of $P_{t}$ is denoted as $P L_{\mathbf{v}_{t a} \mathbf{v}_{t b}}$, then $P_{t}$ is $P_{t_{\text {shonest }}}$ only if $P L_{\mathbf{v}_{t a} \mathbf{v}_{t b}}<\frac{T L}{2}$ where $T L$ is the total path length (i.e., perimeter) of the polygon.

$P_{t_{-} \text {shorest }}$ is further classified into two types: Positive path $P^{+}$and negative path $P^{-}$. If we denote the set of points on the polygons that are nearest to the end points of all façades of the same building (or polygon) other than $f_{t}$ as $\mathbf{K}_{t}$ (i.e., $\mathbf{K}_{t}$ contains points nearest to the endpoints of façades $f_{r^{\prime}=1, \ldots t-1, t+1, \ldots, s}$ such that $\left.r^{\prime} \cup t=s\right)$, then $P_{t_{-} \text {shortest }}$ of the reconstructed façade $f_{t}$ is defined to be $P^{+}$if set of points $\mathbf{P}_{t_{-} \text {shortest }}$ belonging to the path $P_{t_{-} \text {shortest }}$ does not contain any element of $\mathbf{K}_{t}$ i.e., $\mathbf{P}_{t_{-} \text {shortest }} \cap \mathbf{K}_{t}=\varnothing$. Thus all façades whose paths are identified as positives are incorporated in the fusion process while façades having negative paths are not considered any further. provides the complete procedure to incorporate façades of both types with the refined building polygon. Steps 12 to 15 in Table 2 pose a condition $C 1$ for all façades of type I such that they do not take part in the fusion process if the change in area of the polygon after incorporating the particular façade is greater than a certain fraction $a_{f}$ of the previous polygon area. $a_{f}$ is fixed to 0.15 in this work. This is to ensure that façades belonging to the inner structures of the building do not interfere during the fusion procedure or in other words only façades that are exterior and define the building outlines are utilized.

Given: Refined polygon of $j$ th building $\tilde{\mathbf{V}}_{j} \& s$ reconstructed

façades $f_{r=1, \ldots, s}$ belonging to the same $j$ th building

1. $\mathbf{V}_{\text {prev }}=\tilde{\mathbf{V}}_{j}$

2. for $t=1$ to $s$ (i.e., total number of reconstructed façades associated to this building polygon)

3. Determine points $\mathbf{v}_{t a}$ and $\mathbf{v}_{t b}$ by computing points on the polygon that are nearest to the two endpoints of the façade $f_{t}$

4. Build a matrix $\mathbf{K}_{t}$ specific to the façade $f_{t}$ that contains points on the polygon that are nearest to the endpoints of all other façades

5. Determine $P_{t \_ \text {shortest }}$

6. if $P_{t_{-} \text {shortest }}$ is also $P^{+}$

7. if façade is identified as type I

8. Compute midpoint of the line segment $L_{t}$ formed by connecting the two points $\mathbf{v}_{t a}$ and $\mathbf{v}_{t b}$

9. Determine two new points $\mathbf{v}_{t a}^{\prime}$ and $\mathbf{v}_{t b}^{\prime}$ by projecting $\mathbf{v}_{t a}$ and $\mathbf{v}_{t b}$ onto another line parallel to the respective façade $f_{t}$ but passing through the midpoint of $L_{t}$

10. Replace them (i.e., vertices of $\mathbf{V}_{\text {prev }}$ within $P_{t_{-} \text {shortest }}$ ) by $\mathbf{v}_{t a}^{\prime}$ and $\mathbf{v}_{t b}^{\prime}$ and store the result in the matrix $\mathbf{V}_{\text {new }}$

11. Compute area of old $\mathbf{V}_{\text {prev }}$ and new $\mathbf{V}_{\text {new }}$ polygons denoted as $A_{\mathbf{V}_{\text {prev }}}$ and $A_{\mathbf{V}_{\text {new }}}$ respectively

12. Check the condition $C 1:\left|A_{\mathbf{V}_{\text {prev }}}-A_{\mathbf{V}_{\text {new }}}\right| \leq a_{f} A_{\mathbf{V}_{\text {prev }}}$

13. if $C 1$ is satisfied

14. $\quad \mathbf{V}_{\text {prev }}=\mathbf{V}_{\text {new }}$

15. end if

16. else

17. Assign $\mathbf{v}_{t a}^{\prime}$ and $\mathbf{v}_{t b}^{\prime}$ to the respective endpoints of the reconstructed façade

18. Replace vertices of $\mathbf{V}_{\text {prev }}$ within $P_{t_{-} \text {shortest }}$ by $\mathbf{v}_{t a}^{\prime}$ and $\mathbf{v}_{t b}^{\prime} \&$ store the result in the matrix $\mathbf{V}_{\text {new }}$

19. $\quad \mathbf{V}_{\text {prev }}=\mathbf{V}_{\text {new }}$

20. end

21. end if

22. end loop

Table 2: Procedure to fuse façades of both types.

Note that there lies some differences in computation of $\mathbf{v}_{t a}^{\prime}$ and $\mathbf{v}_{t b}^{\prime}$ for type I and II façades (steps 8-9 and 17 in ). The reason 
for this is due to the fact that point density on building roofs is quite varying and can contain gaps in between. This could lead to under reconstruct the building footprint i.e., part of the building roof region could not be reconstructed due to unavailability of points. Presence of type II façades implicitly validates this plausible phenomenon and therefore fusion of refined polygons by fully incorporating the reconstructed façades (of type II only) ) result in improved overall accuracy of reconstruction. Doing same for type I façade, on the other hand, may affect the footprint polygon in presence of façades belonging to inner building structures. Thus, only the orientation of type I façade is essentially incorporated by the proposed procedure (steps 8-9 in ). In addition to this, steps 12 to 15 in also pose a condition $C 1$ for type I façades such that they do not take part in the fusion process if the change in area of the polygon after incorporating the particular façade is greater than the certain fraction $a_{f}$ (fixed to 0.15 in this work) of the previous polygonal area. Thus, using condition $C 1$ together with the method of type I façade fusion, it is ensured that façades belonging to the inner structures of the building do not interfere during the fusion procedure or in other words only façades that are exterior and define the building outlines are utilized.

\subsection{Identification of rectilinear buildings}

The next step in the reconstruction procedure is to identify if the building is composed of two or more than two dominant directions. In case the building polygons is composed of only two dominant directions orthogonal to each other, rectilinear constraints are then added to derive geometrically correct and better visually looking building shapes.

The decision of identifying a rectilinear building is based on its estimated dominant/principal direction. For this purpose, the principal direction of the case 2 building polygons (i.e., having one or more reconstructed façades associated to each polygon) is easily determined by assigning it to the direction vector computed by subtracting the endpoints of the longest reconstructed façade paired to it. For case 3 building polygons, the principal direction is directly estimated from the polygon itself. Since no façade is associated to them, a weighted method based on polygonal edge lengths is employed to estimate the two orthogonal principal directions of the building. The basic idea is to give weight to each edge of the polygon according to its relative length (with respect to total polygon length) and the angular deviation which it makes with a particular direction vector $\mathbf{d v _ { q }} \cdot \mathbf{d \mathbf { v } _ { q }}$ is candidate for one of the two orthogonal principal directions and is rotated within a certain interval to minimize the following objective function (Zhang et al., 2006):

$$
P D=\sum_{i=1}^{n} g_{1}\left(l_{i}\right) g_{2}\left(\varphi_{i}\left(\beta_{i}, \phi_{\mathbf{d v}_{q}}\right)\right)
$$

where $n$ is the total number of vertices of the polygon and $\beta_{i}\left(0 \leq \beta_{i} \leq 90^{\circ}\right)$ is the angular deviation of each edge $l_{i}$ with respect to a particular direction vector $\mathbf{d v}_{q} \cdot \beta_{i}$ is computed similar to (1) with the difference that the two direction vectors are $\mathbf{d v}_{j}$ and $\mathbf{d v}_{q}$ instead of direction vectors of consecutive edges $\mathbf{d v}_{j}$ and $\mathbf{d v}_{j+1} . \phi_{\mathbf{d v}_{q}}$ is anticlockwise rotation angle which $\mathbf{d} \mathbf{v}_{q}$ makes with the unrotated coordinate system. $\varphi_{i}(\cdot)$ is a function that maps the angular deviations $\beta_{i}$ to one of the two orthogonal directions (or axes) as defined by direction vector $\mathbf{d v}_{q}$ and its corresponding normal vector. It is computed as

$$
\varphi_{i}\left(\beta_{i}, \phi_{\mathbf{d v}_{q}}\right)= \begin{cases}\beta_{i} & \text { if } \beta_{i} \leq 45^{\circ} \\ 90-\beta_{i} & \text { if } \beta_{i}>45^{\circ}\end{cases}
$$

Both $g_{1}(\cdot)$ and $g_{2}(\cdot)$ are the weighting functions. $g_{1}(\cdot)$ assigns weight to each edge based on its relative length with respect to the overall length of the polygon edges. It is constructed in a way such that edges with longer lengths contribute less in (2) as compared to shorter edges lengths. Following linear function is used to describe $g_{1}(\cdot)$ :

$$
g_{1}\left(l_{i}\right)=1-\frac{l_{i}}{\sum_{i=1}^{n} l_{i}}
$$

Similarly, $g_{2}(\cdot)$ assign weights to each edge based on its $\varphi_{i}$ value. Assignment of weight is directly proportional to $\varphi_{i}$ i.e., lower weight is given to an edge with lower $\varphi_{i}$ inferring that edges close to one of the two orthogonal directions are given less weight as compared to the ones that are deviating. Since the span of $\varphi_{i}$ for each edge is defined to be within the interval $\left[045^{\circ}\right]$, therefore $g_{2}(\cdot)$ is computed by adopting the following linear function as:

$$
g_{2}\left(\varphi_{i}\left(\beta_{i}, \phi_{\mathbf{d v}_{q}}\right)\right)=\frac{\varphi_{i}}{45}
$$

Solution of (2) is obtained by rotating $\mathbf{d} \mathbf{v}_{q}$ within the interval $\left[090^{\circ}\right]$. An optimum (or minimum) $\hat{\phi}_{\mathbf{d v}_{q}}$ is found by comparing $P D$ for each $\phi_{\mathbf{d v}_{q}}$. The direction vector and its corresponding normal vector associated to the optimum (or minimum) $\hat{\phi}_{\mathbf{d v}_{q}}\left(=\underset{\phi_{\mathbf{d v}_{q}}}{\arg \min }(P D)\right)$ thus describe the two orthogonal principal directions.

Once the principal/dominant directions are determined, the following procedure is adopted for identification of rectilinear buildings:

1) Determine angular difference $\beta_{i}\left(0 \leq \beta_{i} \leq 90^{\circ}\right)$ of all the edges of the polygon with respect to the dominant/principal directions;

2) Compute histogram of these angular differences;

3) Find the edges whose angular differences are within the bin intervals $\left[\begin{array}{ll}0^{\circ} & 20^{\circ}\end{array}\right]$ and $\left[\begin{array}{ll}70^{\circ} & 90^{\circ}\end{array}\right]$;

Identify the polygon to be rectilinear if the lengths of these edges are more than $0.75 \times T L$ i.e., $75 \%$ of the total polygonal length $T L$.

\subsection{Addition of rectilinear constraints}

Subsequently, rectilinear constraints are added to the identified building polygons to yield much better (visually appealing) geometric building shapes. The following steps are performed to obtain rectilinear building footprint:

1) Classify each edge of the building polygon such that it belongs to one of the two orthogonal principal axes based on its angular deviation (i.e., an edge is associated to that 
principal direction with whom the angular difference is less);

2) Merge all adjacent edges that share a same class, i.e., associated to the same principal direction;

3) Apply rectilinear transformation to every merged polygonal edge by projecting it onto its corresponding principal axis/vector;

4) Computing intersection (or vertex) points between the consecutive vertices.

\section{EXPERIMENTAL RESULTS \& VALIDATION}

\subsection{Dataset}

To validate our approach, we tested the algorithm on the TomoSAR point clouds generated from a stack of 102 TerraSAR-X high resolution spotlight images from ascending orbit using the Tomo-GENESIS software developed at the German Aerospace Center (DLR) (Zhu et al., 2013). The test area contains relatively complex building structures and covers approximately $1.5 \mathrm{~km}^{2}$ in the city of Berlin, Germany. The number of TomoSAR points in the area of interest is about 0.52 million.

\subsection{Reconstruction results}

Figure 2(a) shows the result of applying façade reconstruction procedure and Figure 2(b) shows result of building points extraction. Extracted building points are then spatially segmented to obtain cluster of points such that each cluster represents an individual building. Figure 3(a) depicts the result of spatially clustering extracted building points into individual buildings. The initial coarse outline of each cluster is then determined using alpha shapes algorithm. It provides good initial estimates of building outlines. However, the value of $\alpha$ affects the shape of the initial coarse polygon. Setting a larger $\alpha$ restricts in obtaining concave boundaries whereas lower values may result in smaller boundary polygons that are actually not present. In addition to this, with smaller $\alpha$ it is also possible that the outer and inner polygons share one (or more) common vertex which may result in improper geometry of footprints. To adaptively select an appropriate value of $\alpha$, we initialize $\alpha=5 \mathrm{~m}$ (reasonable trade-off for our data) which is recursively incremented by $1 \mathrm{~m}$ if resulting polygons share common vertices or minimum area of any resulting polygon is less than $50 \mathrm{~m}^{2}$. Black polygons in Figure 3(a) surrounding each individual segmented building cluster depict their corresponding alpha polygons. Refinement of the initial coarse alpha vertices is then carried out by computing angular deviation at each vertex point. The threshold value $\theta_{\text {ang }}=20^{\circ}$ is used which consequently remove all vertices having angular deviations less than $20^{\circ}$ from their adjacent neighboring vertices. $\theta_{\text {ang }}=0$ results in the original alpha polygons i.e., no refinement or regularization. Setting a too high value for $\theta_{\text {ang }}$ may however result in over refinement/smoothing. Refined or smoothed alpha polygons are then fused with the reconstructed façades. Later, rectilinear constraints are added to the building polygons that are identified to be rectilinear. Figure 3(b)-(d) depicts the results of building footprint reconstruction.

\subsection{Reference footprints}

The actual ground truth data are missing for exact quantitative evaluation of the approach. In order to provide some qualitative measures of the algorithm performance, we compared our building extraction results to reference polygons downloaded from the OpenStreetMap (OSM) (“GEOFABRIK,” 2015).

\subsection{Evaluation strategy}

In order to evaluate the reconstruction results, we rasterized both the reconstructed and reference polygonal footprints onto an image with pixel resolution of $1 \mathrm{~m}$ (i.e., 1 pixel corresponds to $1 \mathrm{~m}^{2}$ spatial area). A difference image created by subtracting the reconstructed footprint image from the reference footprint image is then used to compute the commission and omission errors as follows:

$$
\begin{aligned}
& \text { Commission error }(\%)=\frac{F_{N}}{A_{r e f}} \times 100 \\
& \text { Omission error }(\%)=\frac{F_{P}}{A_{r e f}} \times 100
\end{aligned}
$$

where $A_{\text {ref }}$ is the area of the reference polygons while $F_{N}$ and $F_{p}$ are number of pixels in the difference image having values of -1 and 1 respectively.

Figure 4 presents the common and difference images. The red pixels in the difference images indicate the building regions that are not reconstructed by the proposed algorithm contributing to the omission errors whereas blue pixels are over reconstructed regions i.e., pixels not part of the reference footprint image but present in the reconstructed image. lists the commission and omission errors obtained for the reconstructed footprints.

\begin{tabular}{|c|c|c|c|c|}
\hline $\begin{array}{c}\text { Reconstructed } \\
\text { footprints }\end{array}$ & $V_{\text {alpha }}$ & $V_{\text {refined }}$ & $V_{\text {facadefised }}$ & $V_{\text {final }}$ \\
\hline $\begin{array}{c}\text { Errors } \\
\begin{array}{c}\text { Commission error } \\
(\%)\end{array}\end{array}$ & 20.20 & 20.43 & 19.13 & 19.43 \\
\hline $\begin{array}{c}\text { Omission error } \\
(\%)\end{array}$ & 12.31 & 12.84 & 13.24 & 14.57 \\
\hline
\end{tabular}

Table 3: Footprint reconstruction statistics.

Hypothetically, the reconstruction results will be improved with higher density of TomoSAR points because more points would be available for parameter estimation. Numerical experiments also demonstrated that reconstruction accuracy is better for buildings with higher density of roof points. For low density roof regions, the reconstruction accuracy is however restricted due to less number of available points which consequently increases the omissions errors. Further improved model based approach might be helpful in this regard. Additionally, the reconstruction errors between the final and coarse 2-D topologies (polygons) are also varying. Thus there is a tradeoff between high geometrical accuracy and low commission and omission errors. As evident, the best tradeoff is obtained after incorporating façades to the coarser building polygons. However, more visually appealing results are produced after introducing rectangular constraints to the rectilinear buildings.

\section{OUTLOOK \& CONCLUSIONS}

In this paper, we have presented an automatic (parametric) approach that only utilized unstructured spaceborne TomoSAR point clouds from one viewing angle to reconstruct building footprints. The approach is modular and systematic. It allows a robust reconstruction of both tall and low buildings, and hence is well suited for monitoring of larger urban areas from space. Moreover, the approach is completely data driven and imposes no restrictions on the shape of the building i.e., any arbitrarily 
shaped footprint could be reconstructed. Also, the approach utilizes roof points in determining the complete shape of the buildings and therefore resolves problems, as mentioned in (Shahzad and Zhu, 2015a), related to the visibility of the façades mainly pointing towards the azimuth direction of the SAR sensor. However, few points still need to be addressed. For instance, the reconstruction accuracy is restricted due to less number of available points and data gaps in the TomoSAR point cloud. This could be improved by incorporating data from other viewing angles and/or adding more constraints such as parallelism or using a model based approaches based on a library of low level feature sets. Also, we have compared our results to the OSM data which is regularly updated but not yet fully complete. Therefore, a more accurate ground truth would be needed for assessing the exact performance of the approach. Nevertheless, this paper presents the first demonstration of automatic reconstruction of 2-D/3-D building footprints from this class of data. Moreover, the developed methods are not strictly applicable to TomoSAR point clouds only but are also applicable to work on unstructured 3-D point clouds generated from a different sensor with similar configuration (i.e., oblique geometry) with both low and high point densities. In the future, we will explore the potential of extending the algorithm towards generation of automatically reconstructed complete watertight prismatic (or polyhedral) 3-D/4-D building models from space.

\section{REFERENCES}

D’Hondt, O., Guillaso, S., Hellwich, O., 2013. Geometric primitive extraction for 3D reconstruction of urban areas from tomographic SAR data, Joint Urban Remote Sensing Event (JURSE), Sao Paolo, Brazil, pp. 206-209.

Dorninger, P., Pfeifer, N., 2008. A Comprehensive Automated 3D Approach for Building Extraction, Reconstruction, and Regularization from Airborne Laser Scanning Point Clouds. Sensors, 8(11), pp. 7323 7343.

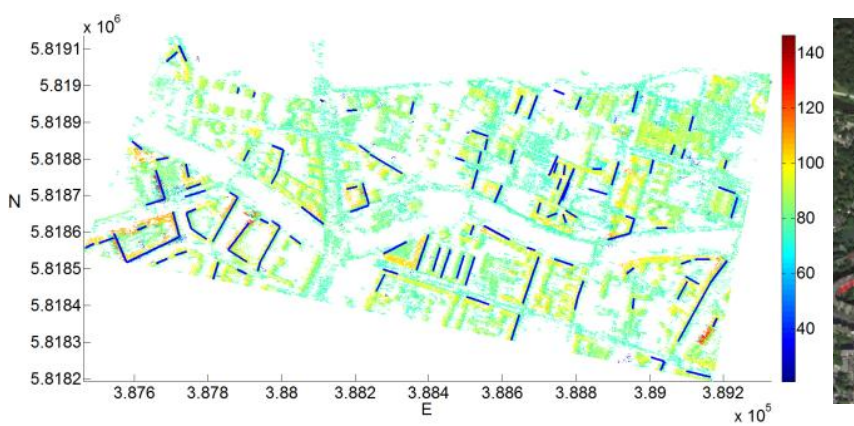

(a)

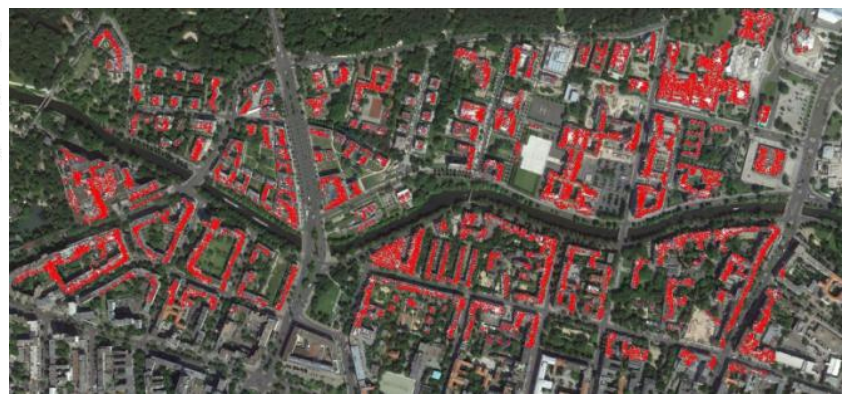

(b)

Figure 2: Results of building extraction: (a) Top view of the TomoSAR points in UTM coordinates of the area of interest in Berlin, Germany. Blue lines depict the reconstructed façade segments (longer than 10 meters). Height of TomoSAR points is color-coded [unit: m]; (b) Extracted building points using approach presented in (Shahzad and Zhu, 2015b) overlaid onto optical image ${ }^{()}$

Google.

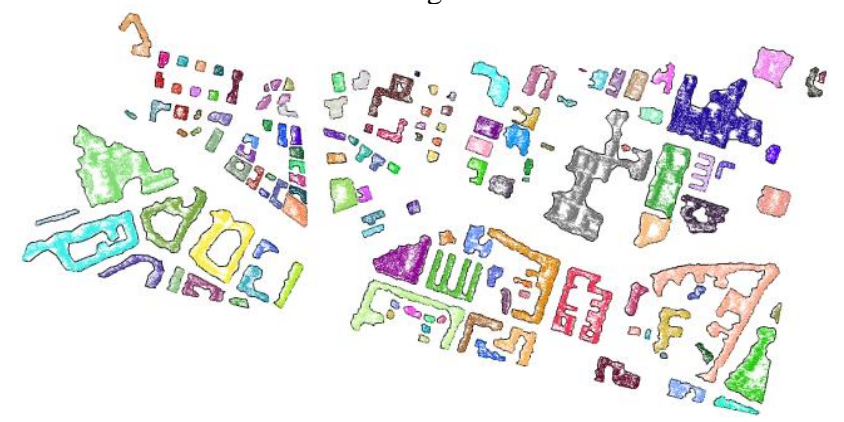

(a) 

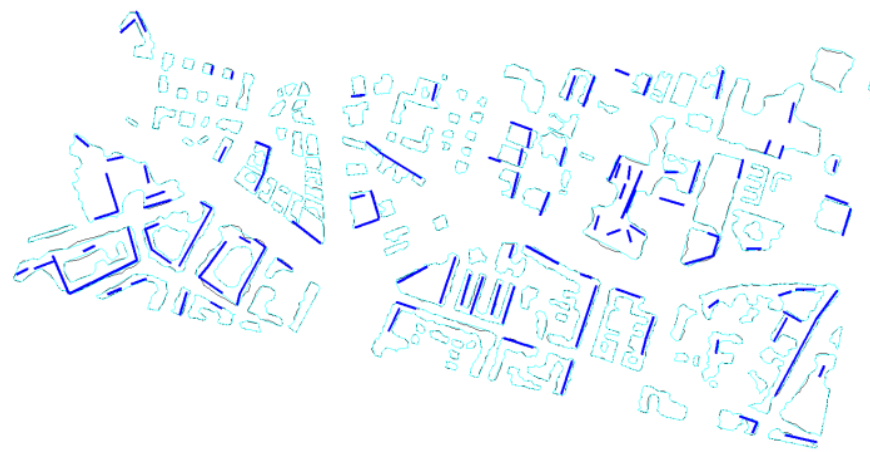

(b)
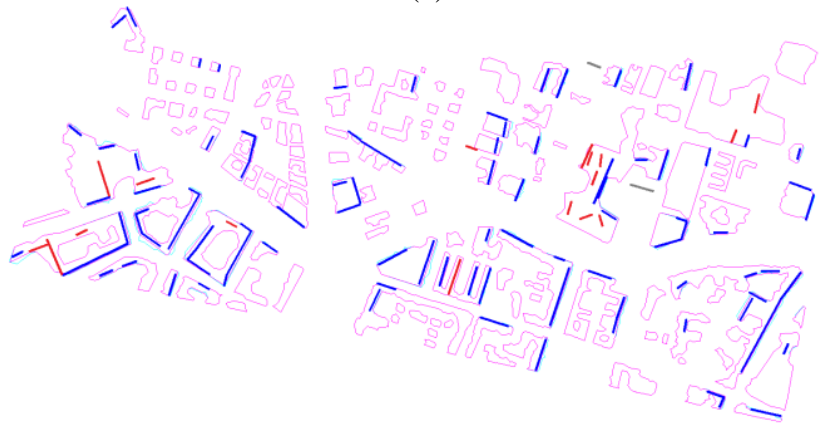

(c)
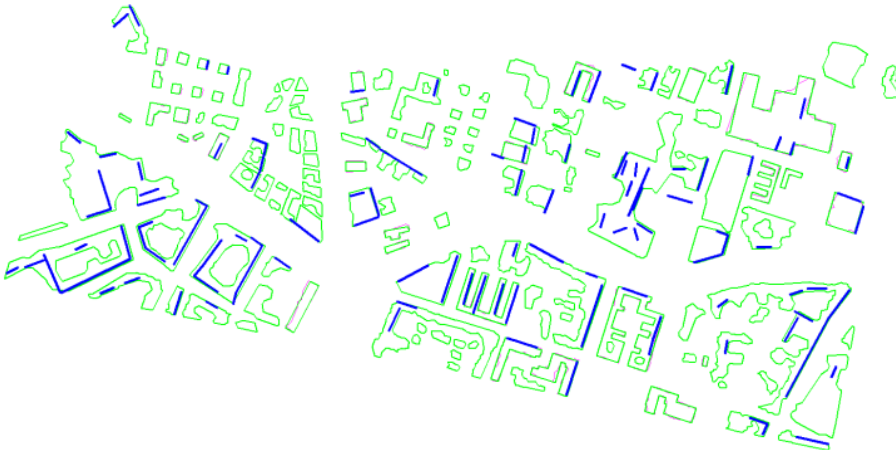

(d)

Figure 3: Footprint reconstruction results: (a) Clustered (or segmented) building points. Black polygons $V_{\text {alpha }}$ surrounding individual segmented building points are the initial coarse boundary/outline obtained using alpha shapes algorithm; (b) Refined (cyan) polygons $V_{\text {refined }}$ obtained after applying recursive angular deviation approach together with 2-D reconstructed façades depicted in blue are overlaid onto alpha shape polygons $V_{\text {alpha }}$; (c) Façades are then incorporated to the refined polygons from (b) depicted in magenta color, symbolized as $V_{f a}$ Façades either identified as $P^{-}$or having condition $C 1$ in not satisfied depicted in red while façades not associated to any building polygon (i.e., case 1) are depicted in gray. Both red and gray façades are not utilized during the Façade-Polygon fusion process; (d) Final rectilinearized polygons $V_{\text {final }}$ obtained after adding rectilinear constraints.
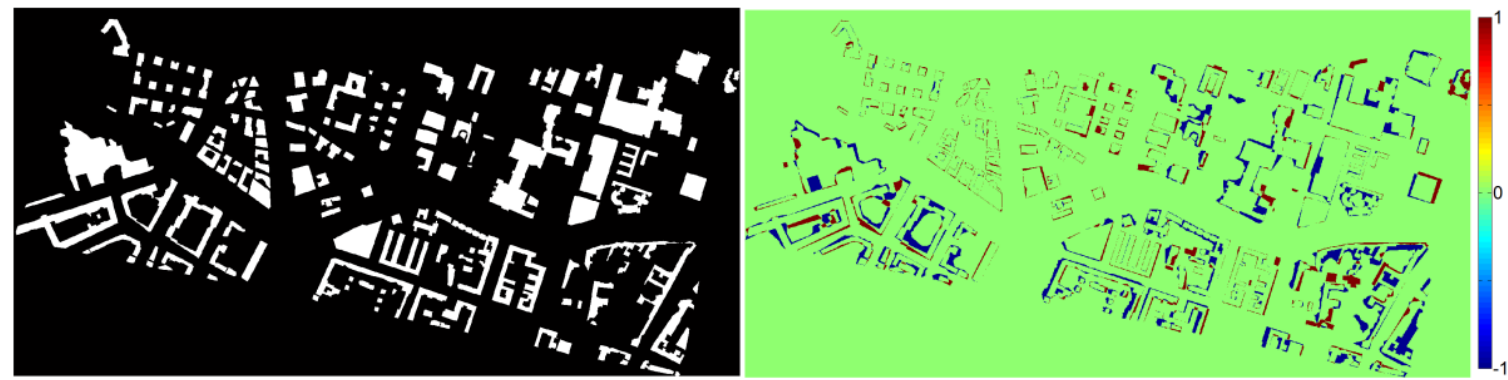

Figure 4: Common (left) and difference (right) images computed using reference footprint image and final reconstructed footprints. The difference image is computed by subtracting the final reconstructed footprints image from the reference footprints image. 